\section{Large-Bowel Obstruction: A Complication of Percutaneous Endoscopic Gastrostomy}

We report here the case of a 57-year-old man who was rushed to the Surgical Department at Cologne University Hospital after trying to commit suicide by cutting his throat. After closing the pharynx defect by direct suture, oral nutrition could not be started due to neurogenic dysphagia. A percutaneous endoscopic gastrostomy (PEG) tube was therefore implanted. The PEG was removed four weeks after implantation by cutting the tube close to the skin. When it was removed, the inner part of the PEG remained in the stomach, and was intended to be eliminated through the gastrointestinal tract. Two weeks later, the patient developed clinical symptoms of mechanical bowel obstruction (Figure 1, 2). Laparotomy and examination of the colon revealed an inflammatory tumor of the sigmoid at the level of the gastrostomy tube inner bumper. The affected segment of the sigmoid was resected (Figure 3). Histological analysis of the tissue showed an ischemic colopathy of unclear genesis, which was reinforced by a local effect of the PEG material, without any sign of malignancy.

In 1991. Waxman reported the first case of bowel obstruction caused by a PEG tube lodged in the terminal ileum (1). Laparotomy was required to remove the inner bumper and relieved the obstruction. A comparative case was observed in 1994 by Mutabagnani (2). A large bowel obstruction after cutting the PEG tube, as described in our case, has not been reported as a complication after PEG removal in the English-language literature. Partial removal of the external parts of the tube, leaving only the tube plate in the stomach, is a subject of controversy in the literature. Pfeil claims always to remove the PEG tube endoscopically, because even in patients who have not had previous abdominal operations, a stenosis caused by inflammatory changes represents a potential obstacle to a foreign body (3). Other authors, by contrast, see lower complication rates, and therefore no indication for endoscopic removal, which is time-consuming and more expensive than cutting the tube (4).

\section{S. P. Mönig H. Hchäfer M. Walter ', K. E. Rehm}

Dept. of Trauma, Hand and Reconstructive Surgery, Cologne University Hospital, Cologne, Germany

2 Dept, of Abdominal. Thoracic, and Vascular Surgery, Cologne University Hospital, Cologne, Germany

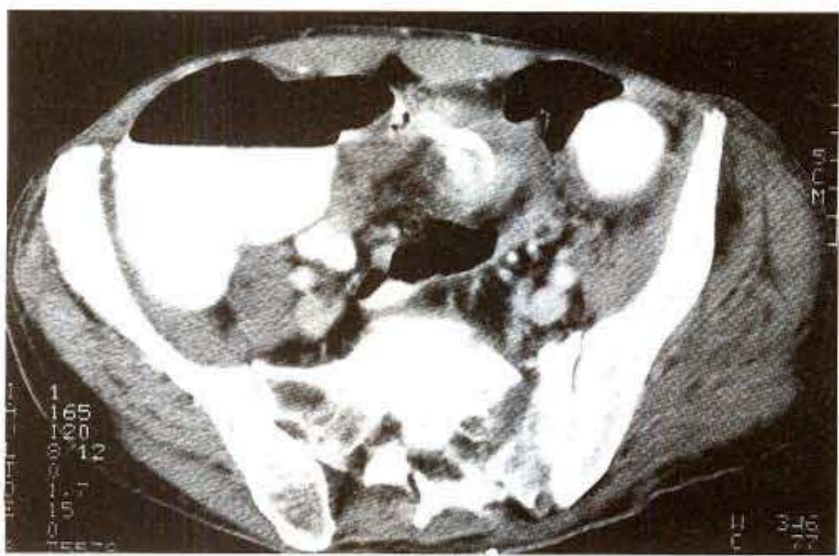

Figure 1: CT of the abdomen, showing the inner bumper of the PEG in the colon.

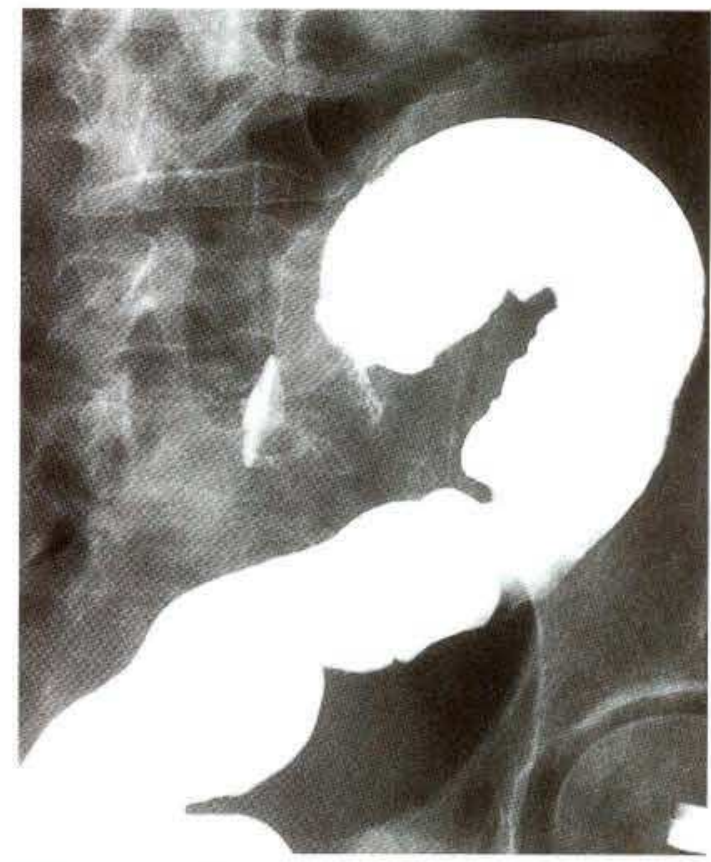

Figure 2: Contrast study of the abdomen, showing an obstruction of the sigmoid at the level of the PEG inner bumper.

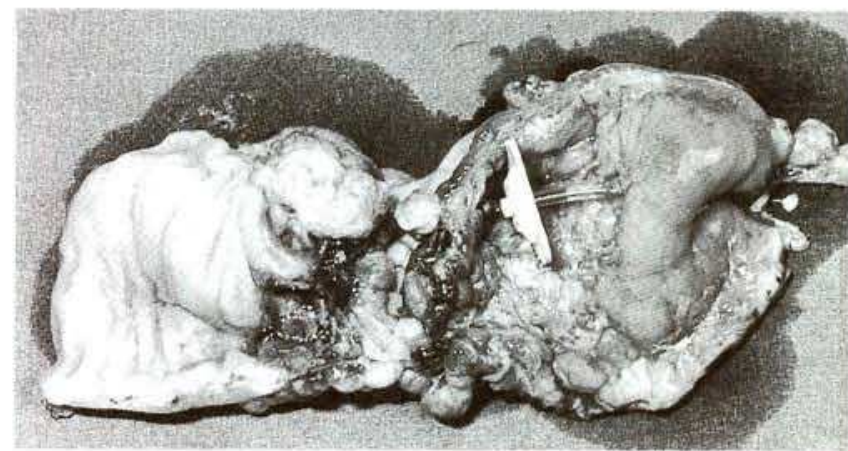

Figure 3: Resected segment of the sigmoid, with the PEG inner bumper

\section{References}

1. Waxman I, Al-Kawas FH. Bass B, et al. PEG-ileus: a new cause of bowel obstruction. Digest Dis Sci 1991:36: 251-4.

2. Mutabagnani KH. Townsend MC. Arnold MW. PEG ileus: a preventable complication. Surg Endosc 1994: 8: 694-7.

3. Pfeil S, Blades E, Yang P. Complications of percutaneous endoscopic gastrostomy removal [letter]. Gastrointest Endosc $1990 ; 36: 316$.

4. Galat SA, Gerig KD, Porter JA, et al. Management of premature removal of the percutaneous gastrostomy. Am J Surg 1990; 56: $733-6$.

Corresponding Author

S. P. Mönig, M.D

Dept. of Surgery

Cologne University Hospital

Joseph-Stelzmann-Strasse 9

50924 Köln, Germany

Fax: $+49-221-4786258$ 